\title{
Synthesis of PR-/RP-chain-based compliant mechanisms - design of applications exploiting fibre reinforced material characteristics
}

\author{
U. Hanke ${ }^{1}$, E.-C. Lovasz ${ }^{2}$, M. Zichner ${ }^{1}$, N. Modler ${ }^{1}$, A. Comsa ${ }^{1}$, and K.-H. Modler ${ }^{1}$ \\ ${ }^{1}$ Faculty of Mechanical Engineering and Machine Science, TU Dresden, Dresden, Germany \\ ${ }^{2}$ Department of Mechatronics, Politehnica University of Timisoara, Timisoara, Romania
}

Correspondence to: U. Hanke (uwe.hanke@tu-dresden.de)

Received: 28 November 2014 - Revised: 18 May 2015 - Accepted: 8 July 2015 - Published: 25 August 2015

\begin{abstract}
Compliant mechanisms have several advantages, especially their smaller number of elements and therefore less movable joints. The flexural members furthermore allow an integration of special functions like balancing or locking. Synthesis methods based on the rigid body model (Howell, 2001; Sönmezv, 2008) or topology optimisation (Zhou and Mandala, 2012) provide practical applications from the advantages of compliant elements. Beside these methods, a much simpler approach is the geometric-based synthesis (Ehlig et al., 2013) which is focused on solving guidance tasks by using RR-chain ${ }^{1}$-based compliant linkages. More compact compliant linkages can be build up by using only $\mathrm{PR}^{2}$ or $\mathrm{RP}^{3}$ chains. Therefore a tool is needed to extend the RR-chain-based approach. The necessary analysis of the compliant beam element can be done by numerical analysis and through experiments. Due to the validity of the Bernoulli beam model the elastic similitude can be specialised and a more general synthesis of compliant beam elements can be created. Altogether a generalised synthesis method can be created for handling different linkage structures as well integrating beam elements derived numerically or by measurement. The advances in this method are applied in the synthesis for a cupholder mechanism made of fiber reinforced material.
\end{abstract}

\section{Introduction}

Classical linkage structures need additional elements (e.g. springs) for balancing or locking, which gives rise to the structure complexity. These features can be directly implemented by using compliant linkages. This function integration used in a compliant cupholder mechanism (Ehlig et al., 2013) lead to a simple two part assembly (RR chain coupled to a beam element $(B)$ : RRB mechanism; Fig. 1). The integrated compliant section allows for the implementation of locking so that additional springs are not required. The applied geometric-based synthesis method allows for its direct

\footnotetext{
${ }^{1}$ one link with two rotational joints $(\mathrm{R})$

2 one link with one frame fixed prismatic joint $(\mathrm{P})$ and one moving rotational joint $(\mathrm{R})$

3 one link with one frame fixed rotational joint $(\mathrm{R})$ and one moving prismatic joint $(\mathrm{P})$
}

use in the design process, providing maximum design freedom regarding position and size of the mechanism.

Much simpler mechanisms can be derived by introducing $P$ joints. The intermediate link can be completely eliminated. Hence for assembling such near monolithic mechanism only one bolt ( $\mathrm{R}$ joint) is needed. To make use of this advantage, the synthesis of compliant mechanisms has to be extended to $P$ joints. The advantage of the geometric method should form the basis when implementing RP/PR chains.

Specialising the similitude theory provides additional benefits in deriving a set of compliant beam elements and in the direct use of experimental data for the mechanism synthesis.

\section{Synthesis}

The compliant beam section $B_{0 \mathrm{~B}}$ of the mechanisms shown in Fig. 1c is integrated in the guidance link $A B$ and acts like a spring element. For the synthesis model, the large deflections 
(a)

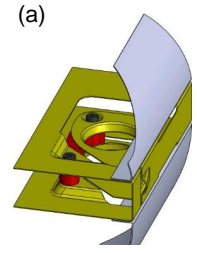

(b)

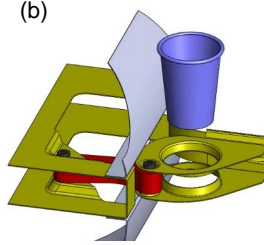

(c)

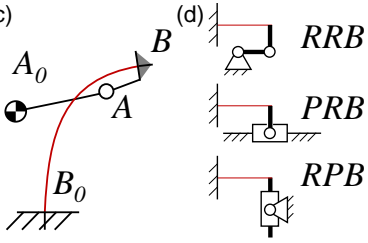

Figure 1. Compliant cupholder mechanism (Ehlig et al., 2013): (a) closed position (b) open position, (c) kinematic scheme of the RRB mechanism, (d) RRB/PRB and RPB mechanism.

of this beam section are introduced by a force/moment load, defined through the coupled joint pair. This can be an RR, PR or RP joint pair. The direct implementation into a graphical synthesis method is problematic. By using the elastic similitude modified for the case of the Bernoulli beam model, a graphical implementation is possible. The result is a synthesis method with two serial coupled synthesis steps (Ehlig et al., 2013):

- synthesis of the RR, PR and RP chains and

- analysis or experiment of the compliant beam element for different load conditions coupled by a similarity transformation for one solution of this set.

The synthesis of the RR, PR and RP chains defines the load configuration of the beam element. For the kinematic solution of the beam element, two parameters can be used to define the load case. These parameters are direct coupled to the scaling and orientation of the beam element.

\subsection{Graphical two-pose synthesis of PR and RP chains}

$\mathrm{RR}, \mathrm{PR}$ and RP chains are link systems with three links: $a$, $b, f$. The following example will include $f$ as frame link, $a$ as guidance link and $b$ as intermediate link. The graphical algorithm used to solve the two-pose task is the construction of the relative pole triangle for a given vertex of this triangle (Luck and Modler, 1990; McCarthy, 2000). In the case of an RR chain, three parameters can be arbitrarily chosen. The triangle of the PR and RP chains degenerates into a triangle with two parallel sides and one vertex at infinity. Therefore the synthesis of these special chains reduces to two parameters. By introducing the rules (see Appendix A), the two-pose synthesis of the PR and RP chains can be completed through the synthesis steps listed in Table 1 referenced in Fig. 2.

The scheme in Fig. 2 is statically balanced in position 1, when the force action line between link $a$ and $f$ is equal to link line $b_{1}$. This fact is of fundamental importance for the implementation of the compliant beam element. The shortest distance to $b_{1}$ measured from $P_{12}^{a f}$ is given by vector $\boldsymbol{r}$.

\subsection{Synthesis of the compliant beam element using the elastic similitude}

The deflection of the compliant beam depends on the material parameters (Young's modulus $E$ ), geometric parameters (the cross section with the area moment of inertia $I$ and beam length $L$ ) and the load condition (force $F$, force direction $\Phi$ and distance $d$ ).

The mathematical description of large deflection behaviour of cantilevered beams is the subject of several scientific publications (Bisshopp and Drucker, 1943; Venanzi et al., 2005; Kimball, 2002; Campanile and Hasse, 2008; Zhang and Chen, 2013; De Bona and Zelenika, 1997). The following statements and equations are based upon the reasoning of Howell (Bisshopp and Drucker, 1943; Howell, 2001; Zhang and Chen, 2013; Campanile and Hasse, 2008).

The Bernoulli-Euler beam theory is the vital element when dealing with large deflection analysis. It says that for constant material parameter $E, I$ the curvature $\kappa$ is proportional to the internal beam moment:

$\kappa=\frac{\mathrm{d} \vartheta}{\mathrm{d} s}=\frac{M}{E I}$.

A constant internal moment means a constant curvature with radius $r=E I / M$ (Howell, 2001; Modler et al., 2013). This hypothesis is, according to Fig. 3, equal to $d \rightarrow \infty$. For all other cases the internal moment reads $M=-F$. $d+F(x \sin \Phi+y \cos \Phi)$. Hence it makes sense to take the second-order form of Eq. (1) (Holst et al., 2011) and use the energy method (De Bona and Zelenika, 1997) to establish the elliptic integral

$$
\frac{1}{\sqrt{2}} \sqrt{\frac{F}{E I}} L=\int_{\vartheta_{1}=0}^{\vartheta_{2}} \frac{\mathrm{d} \vartheta}{\sqrt{\cos (\Phi+\vartheta)+C}},
$$

where $L$ is the beam length and $C$ an integration constant. The constant $C$ is directly coupled to the initial conditions on the clamped side of the beam: $C=F d^{2} /(2 E I)-\cos \Phi$. For a set of known parameters $F, d, E, I$ the integral (2) can be resolved numerically (Howell, 2001; Campanile and Hasse, 2008; Kimball, 2002; Venanzi et al., 2005).

Using the similitude theory (Kerle, 2006; Weber, 1930) leads to a more general way of handling compliant beam elements. The idea behind this synthesis is the direct implementation of a precalculated or measured beam element. The elastic similitude is defined by the Hooke number:

$H o=\frac{F}{E L^{2}}$.

In this case, a scaling of dimensions affects all dimensions and therefore the section modulus $I$ as well. For the compliant beam element, bending is the major load case. So it is possible to specialise the similitude to beams and use a modified Hooke number: 
Table 1. Synthesis of the RP and PR chains.

\begin{tabular}{|c|c|c|}
\hline Step & PR chain & RP chain \\
\hline 1 & Set moving joint $A_{1}^{a b}$ & Set rotatory ground joint $A_{0}^{b f}$ \\
\hline 2 & Draw line $a_{1}$ defined by joint $A_{1}^{a b}$ and pole $P_{12}^{a f}$ & Draw line $f_{1}$ defined by joint $A_{0}^{b f}$ and pole $P_{12}^{a f}$ \\
\hline 3 & Draw line $f_{1}$ defined by $P_{12}^{a f}, \alpha_{12}^{a f}$ and line $a_{1}$ & Draw line $a_{1}$ defined by $P_{12}^{a f}, \alpha_{12}^{a f}$ and line $f_{1}$ \\
\hline 4 & Draw line $b_{1}$ defined by $A_{1}^{a b}, \alpha_{12}^{a b}=\alpha_{12}^{a f}$ and line $a_{1}$ & Draw line $b_{1}$ defined by $A_{0}^{b f}, \alpha_{12}^{b f}=\alpha_{12}^{a f}$ and line $f_{1}$ \\
\hline 5 & $\begin{array}{l}\text { The direction of translation of the prismatic joint } A_{0}^{b f \infty} \\
\text { is perpendicular to line } b_{1}\end{array}$ & $\begin{array}{l}\text { The direction of translation of the prismatic joint } A_{1}^{a b \infty} \\
\text { is perpendicular to line } b_{1}\end{array}$ \\
\hline
\end{tabular}

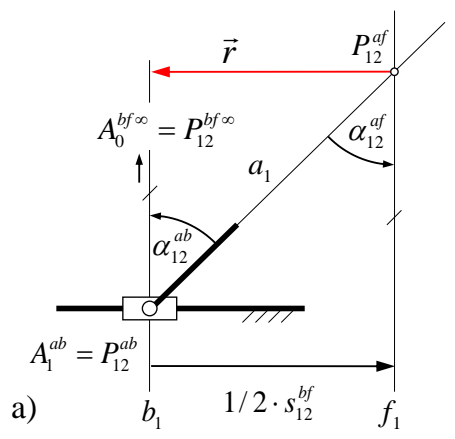

Figure 2. Two-pose synthesis of the (a) PR chain, (b) RP chain.

$m H o=\frac{F L^{2}}{E I}$,

where the section modulus $I$ can be independently scaled. The square root of this number is the left hand side of Eq. (2) (see $\sqrt{\frac{F}{E I}} L=\sqrt{\mathrm{mHo}}$ ). Hence Eq. (2) reads

$\sqrt{\mathrm{mHo}(C)}=\int_{\vartheta_{1}=0}^{\vartheta_{2}} \frac{\mathrm{d} \vartheta}{\sqrt{\cos (\Phi+\vartheta)+C}}$.

Each modified Hooke number $m H o$ is a unique value for a compliant beam dependent on the angles $\vartheta_{1}, \vartheta_{2}$ and the load condition $C$. Therefore deriving a set of deflected beams is described by a set of different $m H o(C)$ as the result of equation (5). The compliant beam is fully defined by naming three of the four variables $F, E, I, L$.

The two positions of the non-deflected and the deflected beam end define the pole of rotation $P_{12}^{a f}$. This pole is coupled to the load condition of the beam. This load condition is defined by two parameters: the load direction $\Phi$ and the distance $d$, defining the moment introduced at the beam end. These parameters define the line $f_{F}$ of the action of the force $F^{a f}$. The shortest distance between the pole $P_{12}^{a f}$ and $f_{F}$ is given by the vector $\boldsymbol{r}$ (Figs. 3a, 4a, b). This vector is used to find the dimensions of the compliant beam element by using a similarity transformation to scale and orient the beam according to the derived PR and RP chain.

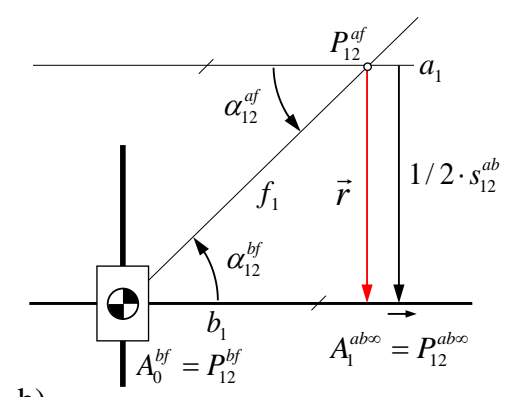

b)

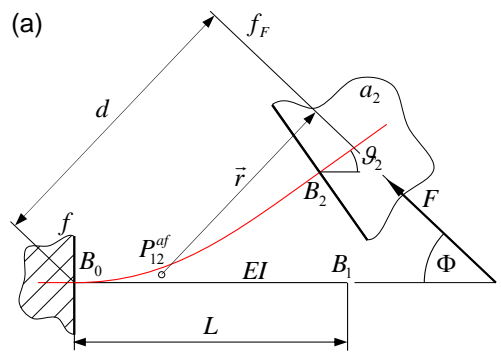

(b)

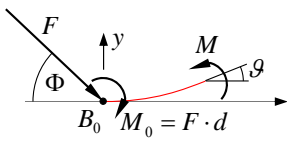

Figure 3. Cantilevered compliant beam under external load: (a) beam model, (b) internal moment $M$.

The elastic similitude makes no difference if the deflections are calculated (Fig. 4a) or measured (Fig. 4b). The rough measurement with the simple test bench still give results useful for further synthesis. Here, a more advanced tool kit (Limaye et al., 2012) could bring more exact results. Errors due to poor beam clamping and friction can be omitted. Doing measurements using a cantilevered beam is a fast and easy way to get to reliable solutions. In this special case the implementation is done by the use of the MATLAB programming environment. The geometric algorithms are based on object-oriented programming with three objects: points, lines and circles with their related methods, and the beam is implemented by solving Eq. (1) numerically as an initial value problem for a normalised beam model. 


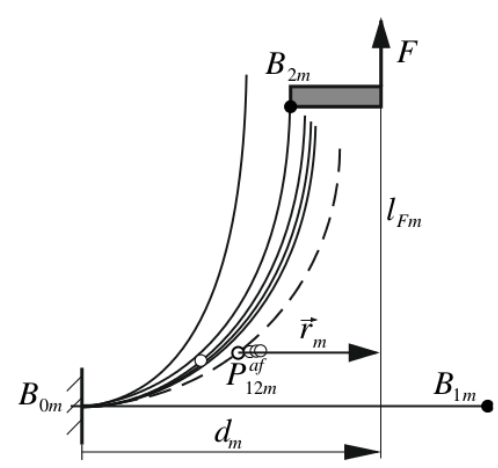

a)

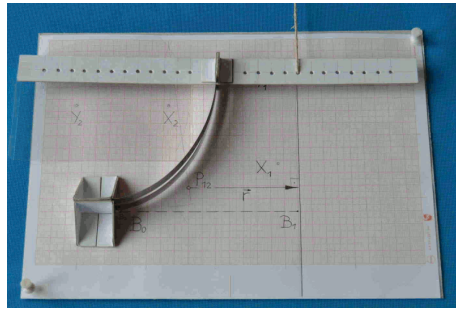

b)

Figure 4. Deflection analysis of a cantilevered beam: (a) calculation for different load sets, (b) measurement using a simple test bench.
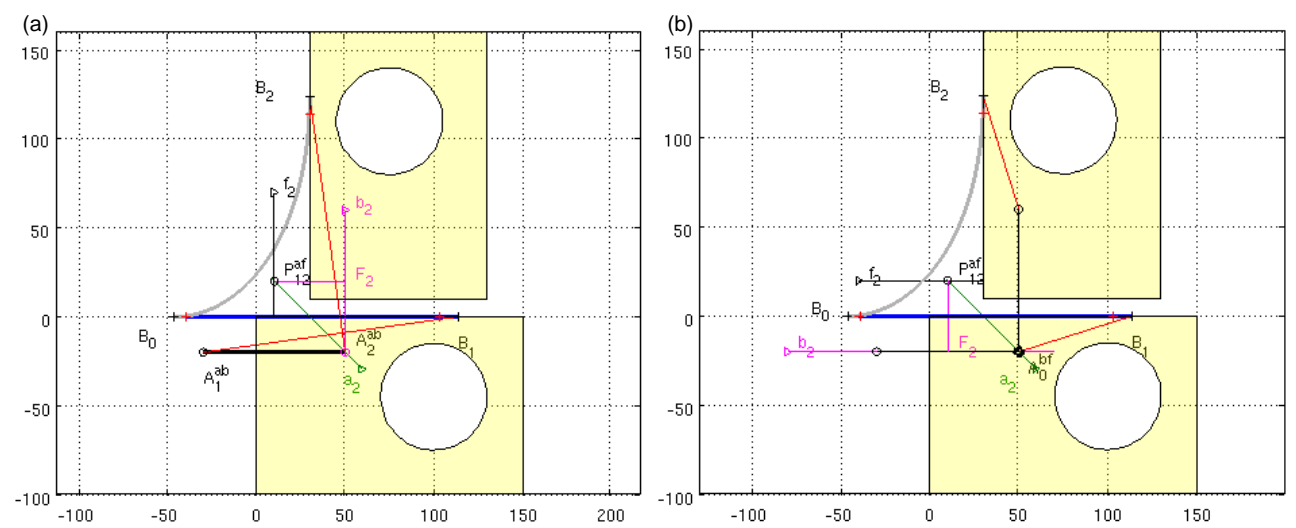

Figure 5. Task solution for compliant: (a) PRB linkage, (b) RPB linkage.

\section{Application}

The described synthesis method is a tool used in designing a cupholder mechanism as one can find in vehicles (Barej et al., 2012). This special application is a feature in luxury cars for holding a cup while driving. It has to be in reaching distance of the driver. When the cupholder is not in use it can be stored/hidden behind the board. We aim to reduce the number of parts via the integration of locking functions within the compliance of the mechanism structure, and we discuss practical differences to the RR-chain-based mechanism shown in Ehlig et al. (2013).

The synthesis task is a two-pose task referring to Ehlig et al. (2013) (Table 2). In the initial position $a_{1}$ the cupholder should be hidden behind the front board. The second position $a_{2}$ offers a panel with a circular hole to place the cup. When the holder is not in use, the front end should be hidden behind the surface of the board. All joints should be located behind the board.

The derived PRB linkage (Prismatic joint, Rotatory joint and compliant Beam element) given in Fig. 5a is characterised by the small distance of the moving joint $A^{a b}$ to the front end of the board. The beam element is relatively
Table 2. Synthesis parameters and results for the RPB and PRB mechanisms.

\begin{tabular}{|c|c|c|c|c|c|c|c|c|}
\hline & $a_{1}$ & $a_{2}$ & $P_{12}^{a f}$ & PR: $A_{1}^{a b}$ & $\begin{array}{l}\mathrm{PR}: A_{2}^{a b} \\
\mathrm{RP}: A_{0}^{b f}\end{array}$ & $B_{0}$ & $B_{1}$ & $B_{2}$ \\
\hline $\mathrm{x}$ & 0.0 & 20.0 & 10.0 & -30.0 & 50.0 & -46.2 & 113.5 & 30.0 \\
\hline $\mathrm{y}$ & 10.0 & 10.0 & 20.0 & -20.0 & -20.0 & -0.0 & -0.0 & 123.5 \\
\hline$\varphi$ & 0.0 & 90.0 & & & & & & \\
\hline
\end{tabular}

large, compared to the RR chain solution given in Ehlig et al. (2013). Extending this distance will enlarge the beam and therefore lead to difficulties in the linkage design and its stability. Thermoplastic fibre reinforced material (FRM) enables the adjustment of its mechanical properties. Material with high strength, coupled with a low Young's modulus $E$ is extremely appropriate for use in compliant structures. The very compact design shown in Fig. 6 is constructible.

The use of thermoplastic matrix material enables easy post-processing by thermal bending. Using preprocessed FRM preforms (Fig. 7a), the final processing in manufacturing this linkage is a three-step folding process (Fig. 7b, c, d) with the final assembly of one bolt. 

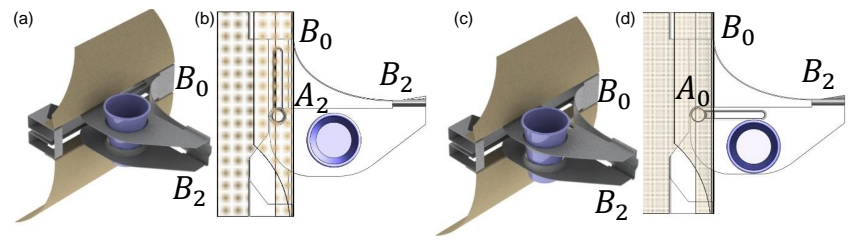

Figure 6. Cupholder linkage: (a) RPB linkage model, (b) RPB linkage transparent top view, (c) PRB linkage model, (d) PRB linkage transparent top view.

As seen in Fig. 5b and Fig. 6c, d the RPB linkage is similar to the PRB linkage discussed. The differences between these solutions are in design and kinematic behaviour. Both linkages are less complex than the RR-chain-based solution given in Ehlig et al. (2013). Instead of two bolts and one link arm, the PRB and RPB linkages only use one bolt to complete the linkage.
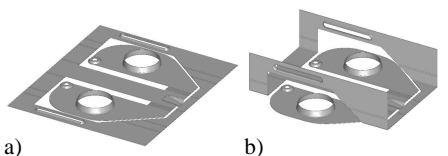

b)

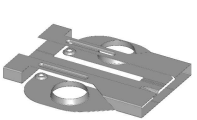

c)

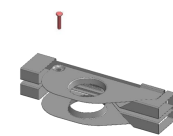

Figure 7. Processing and assembly of a PRB linkage: (a) preform, (b) first folding step, (c) second folding step, (d) bolt assembly.

\section{Conclusions}

Extending the graphical-based synthesis approach using elastic similitude to PR-/RP-chain-based compliant linkages widens the range of compliant linkages to include more compact mechanisms. The drawback is one less synthesis parameter compared to the RRB linkage: two parameters defining the PR/RP chain and one the size of the beam element are three free parameters that allow geometric synthesis for PR-/RP-chain-based mechanisms with a compliant beam element. The extension of the synthesis scheme given in Ehlig et al. (2013) to PR-/RP-chain-based compliant linkages widens the range of compliant linkages to include near monolithic structures. These structures are less complex and characterised by a simplified design. Future research work should focus on coupled beam structures as the BRB linkage as a further step to in designing monolithic mechanisms. 


\section{Appendix A: Index rules}

Using graphical synthesis methods, it is useful to name links with small letters and joints/poles as big letters. Motions are defined by link positions and the reference link from where this movement is measured. In handling definite positions we have to distinguish between link pairs and position pairs. Therefore all kinematic symbols are marked with indices $P_{12}^{a f}$ (af: link index $\rightarrow a$ moves in reference to $f ; 12$ : pose index $\rightarrow$ position 1 moves to position 2). This nomenclature leads to the rules given in Fig. A1.

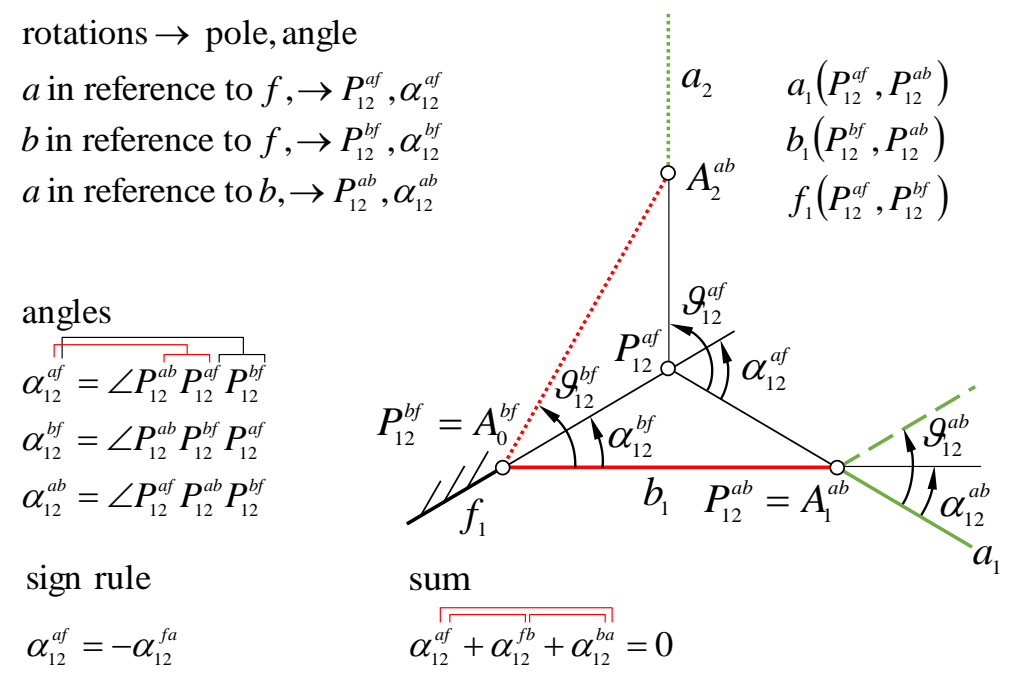

Figure A1. Rules and nomenclature for the graphical synthesis demonstrated at a two-pose RR chain example. 


\section{The Supplement related to this article is available online at doi:10.5194/ms-6-155-2015-supplement.}

Acknowledgement. The authors would like to express their gratitude to the Deutsche Forschungsgemeinschaft (DFG), which supports the research leading to this publication as part of the scope of the subproject D2 of the Collaborative Research Centre SFB 639 "Textile-Reinforced Composite Components in FunctionIntegrating Multi-Material Design for Complex Lightweight Applications".

Edited by: J. Schmiedeler

Reviewed by: two anonymous referees

\section{References}

Barej, M. and Hüsing, M., and Corves, B.: Teaching Mecanism Theory - From Hands-on Analysis to Virtual Modeling, in: New Trends in Mechanism and Machine Science, edited by: Viadero, F. and Ceccarelli, M., Mechanism and Machine Science, 7, 703710, Springer Netherlands, 2001.

Bisshopp, K. E. and Drucker, D. C.: Large Deflection of Cantilever Beams, Q. Appl. Math., 3, 272-275, 1945.

Campanile, L. F. and Hasse, A.: A simple and effective solution of the elastica problem, Proceedings of the Institution of Mechanical Engineers, Part C: J. Mech. Eng. Sci., 222, 2513-2516, 2008.

De Bona, F.; Zelenika, S.: A generalized elastica-type approach to the analysis of large displacements of spring-strips, 211, 509517, Journal of Mechanical Engineering Science, (1997).

Ehlig, J., Hanke, U., Lovasz, E.-C. Zichner, M., and Modler, K.-H.: Geometrical synthesis approach for compliant mechanisms - Design of applications exploiting fibre reinforced material characteristics, in: New Advances in Mechanisms, Transmissions and Applications, edited by: Petuya, V., Pinto, C., and Lovasz, E.C., Mechanisms and Machine Science, 17, 215-224, Springer Netherlands, 2013.
Holst, G. L., Teichert, G. H., and Jensen, B. D.: Modeling and Experiments of Buckling Modes and Deflection of FixedGuided Beams in Compliant Mechanisms, J. Mech. Design, 133, 051002, doi:10.1115/1.4003922, 2011.

Howell, L. 1.: Compliant Mechanisms, Wiley-Interscience, New York, 480 pp., 2001.

Kerle, H.: Zur Entwicklung von Baureihen für Getriebe und von belastbaren Getriebemodellen auf der Grundlage der Ähnlichkeitsmechanik, Bewegungstechnik VDI-Getriebetagung 2006, VDI-Berichte 1966, VDI-Verlag, Düsseldorf, 2006.

Kimball, C. and Tsai, L.-W.: Modeling of Flexural Beams Subjected to Arbitrary End Loads, J. Mech. Design, 124, 223-235, 2002.

Limaye, P., Ramu, G., Pamulapati, S., and Ananthasuresh, G. K.: A compliant mechanism kit with flexible beamsand connectors along with analysis and optimal synthesis procedures, Mech. Mach. Theory, 49, 21-39, 2012.

Luck, K. and Modler, K.-H.: Getriebetechnik - Analyse Synthese Optimierung, Springer-Verlag, Wien New York, 1990.

McCarthy, J. M.: Geometric Design of Linkages, Springer-Verlag, New York, 2000.

Modler, N., Modler, K.-H., Hufenbach, W. A., Jaschinski, J., Zichner, M., Hanke, U., and Ehlig, J.: Optimization of a Test Bench for Testing Compliant Elements Under Shear-Force-Free Bending Load, Procedia Materials Science, 2, 130-136, 2013

Sönmez, Ü. and Tutum, C. C.: A Compliant Bistable Mechanism Design Incorporating Elastica Buckling Beam Theory and Pseudo-Rigid-Body Model, 130, 042304, doi:10.1115/1.2839009, J. Mech. Design, 2008.

Venanzi, S., Giesen, P., and Parenti-Castelli, V.: A novel technique for position analysis of planar compliant mechanisms, Mech. Mach. Theory, 40, 1224-1239, 2005.

Weber, M.: Das Allgemeine Ähnlichkeitsprinzip der Physik und sein Zusammenhang mit der Dimensionslehre und der Modellwissenschaft, Jahrbuch der Schiffbautechnischen Gesellschaft, 31. Bd., Kap. XIV, 274-354, Springer-Verlag, Berlin, 1930.

Zhang, A. and Chen, G.: A Comprehensive Elliptic Integral Solution to the Large Deflection Problems of Thin Beams in Compliant Mechanisms, Journal of Mechanisms and Robotics, 5, 021006, doi:10.1115/1.4023558, 2013

Zhou, H. and Mandala, A. R.: Topology Optimization of Compliant Mechanisms Using the Improved Quadrilateral Discretization Model, Journal of Mechanisms and Robotics, 4, 021007, doi:10.1115/1.4006194, 2012. 